\title{
$7 \mathrm{~dB}$ quadrature squeezing at $860 \mathrm{~nm}$ with periodically-poled $\mathrm{KTiOPO}_{4}$
}

\author{
Shigenari Suzuki, ${ }^{1,2,3}$ Hidehiro Yonezawa, ${ }^{1,4}$ Fumihiko Kannari, ${ }^{3}$ Masahide Sasaki, ${ }^{2,4}$ and Akira Furusawa ${ }^{1,4}$ \\ ${ }^{1}$ Department of Applied Physics, The University of Tokyo, \\ 7-3-1 Hongo, Bunkyo-ku, Tokyo 113-8656, Japan \\ ${ }^{2}$ Advanced Communications Technology Group, National Institute of Information and Communications Technology (NICT), \\ 4-2-1 Nukui-kitamachi, Koganei, Tokyo 184-8795, Japan \\ ${ }^{3}$ Department of Electronics and Electrical Engineering, Keio University, \\ 3-14-1 Hiyoshi, Kohoku-ku, Yokohama 223-8522, Japan \\ ${ }^{4}$ CREST, Japan Science and Technology Agency, 1-9-9 Yaesu, Chuo-ku, Tokyo 103-0028, Japan
}

\begin{abstract}
We observed $-7.2 \pm 0.2 \mathrm{~dB}$ quadrature squeezing at $860 \mathrm{~nm}$ by using a sub-threshold continuouswave pumped optical parametric oscillator with a periodically-poled $\mathrm{KTiOPO}_{4}$ crystal as a nonlinear optical medium. The squeezing level was measured with the phase of homodyne detection locked at the quadrature. The blue light induced infrared absorption was not observed in the experiment.
\end{abstract}

PACS numbers: 03.67.Hk, 42.50.Dv

Squeezed states of optical fields are important resources for photonic quantum information technology particularly with continuous variables $1,2,3,4,5,6$, 7, 8, 9]. The performance of such protocols is limited directly by the squeezing level [1, 10]. For example, the fidelity in $n$ cascaded quantum teleportation of coherent states scales as

$$
F(n, r)=1 /\left(1+n e^{-2 r}\right)
$$

with $r$ the squeezing degree [2, 11, 12]. The amount of information extracted by quantum dense coding must increase as

$$
I\left(n_{s}, r\right)=\ln \left[1+n_{s} e^{2 r}\right]
$$

where $n_{s}$ is the average photon number used for signal modulation [5, 7].

The highest squeezing level observed so far under practical setting with the phase locked was $-6.0 \pm 0.3 \mathrm{~dB}$ by Polzik et al. 13. They employed a continuous-wave (CW) Ti:Sapphire laser at $852 \mathrm{~nm}$ and a sub-threshold degenerate optical parametric oscillator (OPO) with a $\mathrm{KNbO}_{3}$ crystal as a nonlinear optical medium. Since then this scheme has been a sort of standard in squeezing experiments at this wavelength range.

For $\mathrm{KNbO}_{3}$, however, the pump (blue) light induced infrared absorption (BLIIRA) has been known as the limiting factor for attaining higher squeezing. A break-through was brought by Aoki et al. [14], using periodically-poled $\mathrm{KTiOPO}_{4}(\mathrm{PPKTP})$. Although existences of the pump light induced absorption in $\mathrm{KTiOPO}_{4}$ (KTP) and PPKTP crystals had already been reported in pulsed light experiments [15], BLIIRA was not observed at $946 \mathrm{~nm}$ in their $\mathrm{CW}$ experiment 14.

In this letter, we report the higher level of squeezing, $-7.2 \pm 0.2 \mathrm{~dB}$, generated with PPKTP at $860 \mathrm{~nm}$ in CW experiment with the phase locked. This squeezing level opens potential applications to new coding in a single mode bosonic channel and sensing technologies [5, 16, 17]. Particularly, it is expected to beat the Holevo capacity limit if the state purity is improved by reducing the present anti-squeezing level because the squeezing level itself already exceeds the theoretical criterion of $-6.78 \mathrm{~dB}[5]$. The size and performance of photonic quantum circuit will also be improved. For example, in the quantum teleportation of coherent states, either five cascaded processes or a single process with a high fidelity of 0.84 could be performed in principle. Furthermore, the wavelength range corresponds to the $\mathrm{Cs}_{2}$ line $(852 \mathrm{~nm})$, and hence fascinating for applications for controlling Cs atoms with non-classical light.

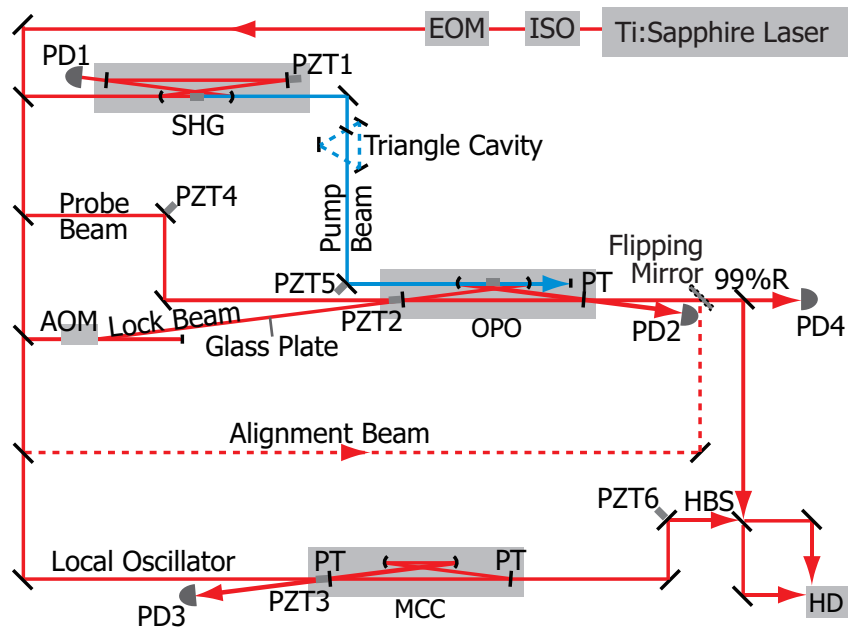

FIG. 1: Experimental setup. ISO: optical isolator, EOM: electro-optic modulator, AOM: acousto-optic modulator, SHG: second harmonic generator (frequency doubler), OPO: sub-threshold degenerate optical parametric oscillator, MCC: mode cleaning cavity, HBS: 50:50 beam-splitter, PTs: partial transmittance mirrors, HD: balanced homodyne detector, PDs: photo-detectors, and PZTs: piezo-electric transducers.

A schematic of our experimental apparatus is shown in Fig. 1. A continuous-wave Ti:Sapphire laser (Coherent MBR-110) at $860 \mathrm{~nm}$ is employed to accomplish this experiment. The beam from the Ti:Sapphire laser is phase-modulated at $15.3 \mathrm{MHz}$ by an electro-optic mod- 
ulator after passing an optical isolator. The modulation is utilized to lock a cavity for frequency doubling and a mode cleaning cavity with conventional FM-sideband locking technique [18].

A part of the beam of around $900 \mathrm{~mW}$ is introduced into the frequency doubler to generate second harmonic at $430 \mathrm{~nm}$ as a pump beam for an OPO. The frequency doubler has a $\mathrm{KNbO}_{3}$ crystal as a nonlinear optical medium in the external cavity with a bow-tie-type ring configuration. An output power from the doubler at 430 $\mathrm{nm}$ is more than $400 \mathrm{~mW}$.

The OPO consists of a bow-tie-type ring cavity and a PPKTP crystal (Raicol Crystals). The cavity has the folding angle of $7^{\circ}$, two spherical mirrors (radius of curvature: $50 \mathrm{~mm}$ ), and two plain mirrors. One of the plain mirrors is a partial transmittance mirror and works as the output coupler while the others are high reflectance mirrors. The round-trip length of $500 \mathrm{~mm}$, the distance between the two spherical mirrors of $58 \mathrm{~mm}$, and the PPKTP crystal (10 $\mathrm{mm}$ long) placed between the spherical mirrors result in waist radii of $20 \mu \mathrm{m}$ inside the crystal and $200 \mu \mathrm{m}$ outside the crystal. The cavity is mechanically stabilized by concatenating mirror mounts of the mirrors with aluminum plates. The OPO easily oscillates with the pump power of $200 \mathrm{~mW}$, while the oscillation threshold $P_{t h}=181 \mathrm{~mW}$ is theoretically obtained from a nonlinear coefficient of the crystal of $E_{N L}=0.023 \mathrm{~W}^{-1}$, an intra-cavity loss $L=0.006$, and a transmittance of the output coupler of $T=0.123$ with the formula $P_{t h}=(T+L)^{2} / 4 E_{N L}$.

The resonant frequency of the OPO cavity is locked using a "lock beam" and a photo-detector (PD2) in Fig. [1 via the conventional FM-sideband locking technique [18]. In order to avoid interference of the lock beam and a "probe beam" in Fig. 11 the beams are in opposite circulations of the cavity. Despite the effort, a fraction of the lock beam circulates backward because of reflection from surfaces of the crystal. This problem is solved by changing the transverse mode and the frequency of the lock beam [13]. The transverse mode is changed from $\mathrm{TEM}_{00}$ to $\mathrm{TEM}_{10}$ by inserting a glass plate into a spatial half part of the beam. The frequency is shifted by about $-120 \mathrm{MHz}$ with an acousto-optic modulator. Thus the probe beam in $\mathrm{TEM}_{00}$ mode and the lock beam in $\mathrm{TEM}_{10}$ mode resonate simultaneously with the OPO cavity.

The generated squeezed light is combined with a local oscillator (LO) at a 50:50 beam-splitter (HBS) and detected by a balanced homodyne detector (HD) with Si photo-diodes (Hamamatsu S3590-06 with special antireflection coating). The circuit noise level of the homodyne detector at $1 \mathrm{MHz}$ is $-18.5 \mathrm{~dB}$ below the shot noise level with the $\mathrm{LO}$ of $3 \mathrm{~mW}$. An output of the $\mathrm{HD}$ is measured for the sideband component at $1 \mathrm{MHz}$ by a spectrum analyzer (Agilent E4402B). The spectrum analyzer is set to the zero-span mode at $1 \mathrm{MHz}$ with $30 \mathrm{kHz}$ resolution bandwidth and $300 \mathrm{~Hz}$ video bandwidth.

A relative phase between the $\mathrm{LO}$ and the squeezed light is locked by use of the probe beam. The probe is locked in

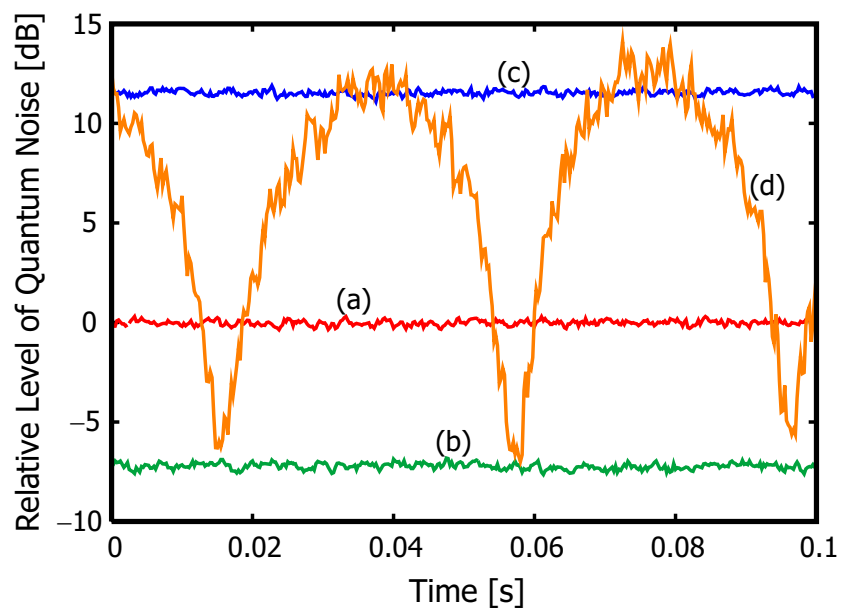

FIG. 2: Power levels of quantum noise. (a) Shot noise level. (b) LO phase is locked at the squeezed quadrature. (c) LO phase is locked at the anti-squeezed quadrature. (d) LO phase is scanned. These are normalized to make the shot noise level $0 \mathrm{~dB}$. All traces except for (d) are averaged 20 times.

phase so that it is minimized/maximized along with the parametric gain of the OPO, and then works as a marker of the squeezed/anti-squeezed quadrature of the squeezed light. As shown in Fig. 1 it is modulated in phase at 64 $\mathrm{kHz}$ by a piezo-electric transducer (PZT4), amplified or deamplified in the OPO, and then detected the fraction of $1 \%$ by a photo-detector (PD4). The phase modulation at $64 \mathrm{kHz}$ is used for two controllers based on the FM-sideband locking technique [18]. An output signal of the PD4 is monitored by one of the controllers which locks the probe via controlling a piezo-electric transducer (PZT5). Then, a relative phase between the probe and the LO is locked by the other controller which monitors an interference signal of these beams from the HD and controls another piezo-electric transducer (PZT6). A fluctuation of the relative phase between the LO and squeezed light is estimated as $\tilde{\theta}=3.9^{\circ}$, which is obtained via measuring rms values of error signals from the control circuits.

In order to improve the homodyne efficiency, the LO is spatially filtered by the mode cleaning cavity which yields the same spatial mode as the OPO output. The overall detection efficiency $\eta=\eta_{P} \eta_{H}$ after the $\mathrm{OPO}$ is obtained from the propagation efficiency of the optical path of $\eta_{P}=0.99$ and the homodyne efficiency $\eta_{H}=0.98$. The homodyne efficiency is dominated by the visibility between the $\mathrm{LO}$ and the OPO output mode because the quantum efficiency of the Si photo-diodes could be treated as unity at the wavelength.

In addition, the alignment beam shown in Fig. 1 is an auxiliary beam which is reserved for use in alignment of constructing the cavity, measuring the intra-cavity loss, and matching the spatial mode of the pump beam with the OPO cavity. For the last application listed above, the alignment beam is converted to the second harmonic 


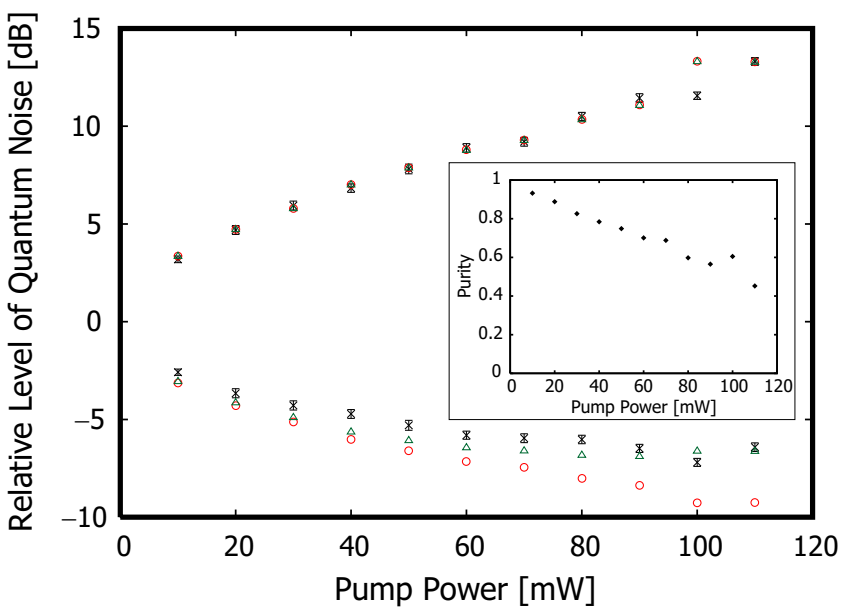

FIG. 3: Squeezing and anti-squeezing levels at several powers of the pump beam. Plots with $\times$ indicate measured values while $\bigcirc$ and $\triangle$ indicate theoretical ones which are calculated from measured classical parametric gains. The phase fluctuation of the LO is taken into account for the plots with $\triangle$ while it is not done for those with $\bigcirc$. The inset shows purities calculated from the observed squeezing and anti-squeezing levels using the definition $\operatorname{Tr}\left\{\hat{\rho}^{2}\right\}$ where $\hat{\rho}$ denotes the density operator of the observed state.

with the OPO as a reference beam for the alignment 13 . The reference beam propagates in the opposite direction to the pump beam and represents the OPO cavity mode. By matching the spatial mode of the reference beam with that of the pump beam, the pump beam is matched with the OPO cavity mode. In order to attain it, a triangle cavity in the path of the pump beam is utilized [13].

Observed quantum noise levels of the OPO output are shown in Fig. 2] They were observed when the pump power was $100 \mathrm{~mW}$. We succeeded in locking the LO phase at the squeezed and anti-squeezed quadratures and in obtaining $-7.2 \pm 0.2 \mathrm{~dB}$ squeezing and $+11.6 \pm 0.2$ $\mathrm{dB}$ anti-squeezing. The squeezing level of the generated state is estimated to be $-7.5 \mathrm{~dB}$ by taking into account the effect of the circuit noise.

Note that the intra-cavity loss $L=0.006$ of the OPO stayed constant independently of the pump beam power, i.e., BLIIRA was not observed. Considering the existence of the pump light induced infrared absorption in experiments with pulsed lasers [15], we infer that the absence of BLIIRA in our experiment is due to the CW operation.

The pump power dependences of the squeezing and anti-squeezing levels are shown in Fig. [3] The observed squeezing level saturates while the pump power increases. This fact could be due to the fluctuation $\tilde{\theta}$ which had a large effect on the observed squeezing level through mixing of the highly anti-squeezed quadrature. Taking account of $\tilde{\theta}$, the theoretical squeezing level $R_{-}^{\prime}$ and antisqueezing level $R_{+}^{\prime}$ are calculated as follows [19]:

$$
R_{ \pm}^{\prime} \approx R_{ \pm} \cos ^{2} \tilde{\theta}+R_{\mp} \sin ^{2} \tilde{\theta}
$$

where $R_{ \pm}$is modeled in Refs. [13, 20]. Considering $\tilde{\theta}=3.9^{\circ}$, the theoretical results almost agree with the experimental ones as shown in Fig. 3 Assuming $\tilde{\theta}=0$, the squeezing level would be $-9.3 \mathrm{~dB}$ with $100 \mathrm{~mW}$ pumping.

In conclusion, we achieved $-7.2 \pm 0.2 \mathrm{~dB}$ quadrature squeezing at $860 \mathrm{~nm}$ with the phase locked at the maximally squeezed quadrature. Moreover, BLIIRA was not observed in a PPKTP crystal for the case of the CW pumped sub-threshold optical parametric oscillator. The resulting state is expected to be utilized to perform various kinds of quantum information processing, to implement precise measurements, and to investigate the photon-atom interactions with Cs atoms.

\section{Acknowledgments}

SS is grateful to Nobuyuki Takei for his experimental support. This work was partly supported by the MPHPT and the MEXT of Japan.

\section{References}

[1] S. L. Braunstein and H. J. Kimble, Phys. Rev. Lett. 80, 869 (1998).

[2] A. Furusawa, J. L. Sørensen, S. L. Braunstein, C. A. Fuchs, H. J. Kimble, and E. S. Polzik, Science 282, 706 (1998).

[3] M. Ban, J. Opt. B: Quantum Semiclassical Opt. 1, L9 (1999).

[4] M. Ban, Phys. Lett. A 276, 213 (2000).

[5] S. L. Braunstein and H. J. Kimble, Phys. Rev. A 61, 042302 (2000).

[6] X. Li, Q. Pan, J. Jing, J. Zhang, C. Xie, and K. Peng, Phys. Rev. Lett. 88, 047904 (2002).

[7] T. C. Ralph and E. H. Huntington, Phys. Rev. A 66,
042321 (2002).

[8] W. P. Bowen, N. Treps, B. C. Buchler, R. Schnabel, T. C. Ralph, H.-A. Bachor, T. Symul, and P. K. Lam, Phys. Rev. A 67, 032302 (2003).

[9] J. Mizuno, K. Wakui, A. Furusawa, and M. Sasaki, Phys. Rev. A 71, 012304 (2005).

[10] N. Takei, H. Yonezawa, T. Aoki, and A. Furusawa, Phys. Rev. Lett. 94, 220502 (2005).

[11] S. L. Braunstein, C. A. Fuchs, and H. J. Kimble, J. Mod. Opt. 47, 267 (2000).

[12] K. Hammerer, M. M. Wolf, E. S. Polzik, and J. I. Cirac, Phys. Rev. Lett. 94, 150503 (2005).

[13] E. S. Polzik, J. Carri, and H. J. Kimble, Appl. Phys. B: Photophys. Laser Chem. 55, 279 (1992).

[14] T. Aoki, G. Takahashi, and A. Furusawa, Opt. Express 14, 6930 (2006). 
[15] S. Wang, V. Pasiskevicius, and F. Laurell, J. Appl. Phys. 96, 2023 (2004).

[16] E. S. Polzik, J. Carri, and H. J. Kimble, Phys. Rev. Lett. 68, 3020 (1992).

[17] C. M. Caves, Phys. Rev. D 23, 1693 (1981).

[18] R. W. P. Drever, J. L. Hall, F. V. Kowalski, J. Hough, G. M. Ford, A. J. Munley, and H. Ward, Appl. Phys. B:
Photophys. Laser Chem. 31, 97 (1983).

[19] T. C. Zhang, K. W. Goh, C. W. Chou, P. Lodahl, and H. J. Kimble, Phys. Rev. A 67, 033802 (2003).

[20] M. J. Collett and C. W. Gardiner, Phys. Rev. A 30, 1386 (1984). 